\title{
La Cadena Productiva de Mora en el Departamento Risaralda en el Marco de un Estudio de Prospectiva a 2032
}

\author{
Blackberry productive value chain in Risaralda within the framework of a prospective \\ study to 2032
}

\author{
L. M. Suárez-Guzmán iD ; D. A. Agudelo-Tapasco iD ; J. W. Zartha-Sossa \\ DOI: https://doi.org/10.22517/23447214.23921 \\ Artículo de investigación científica y tecnológica \\ G. L. Orozco-Mendoza
}

Resumen- El presente artículo, describe las actividades y resultados de investigación del proyecto "ALIANZA REGIONAL PARA EL FORTALECIMIENTO DEL SECTOR AGROINDUSTRIAL EN RISARALDA" - Componente Prospectiva, mediante la aplicación del método Delphi, que consiste en una consulta a un número de expertos o grupos de interés del sector Mora, sobre la base de un cuestionario, que se responde anónimamente en dos rondas. El objetivo del estudio es ofrecer herramientas de decisión a los actores del sector productivo de mora, en temas relacionados con ciencia, tecnología e innovación, los cuales servirán para el fortalecimiento y desarrollo de esta cadena productiva en todos sus eslabones. Como resultados importantes, se resaltan la priorización de 26 temas en 6 agrupadores: mora (cultivo), transformación, logística, comercialización, poscosecha y sostenibilidad, destacándose nueve temas prioritarios para la segunda ronda Delphi: Manejo del cultivo de mora, prácticas de manejo del cultivo de mora, manejo de registros, costos de producción, producción (orgánica o limpia), poscosecha, colorantes, saborizantes, fuentes de financiamiento. Se resalta la participación de expertos internacionales de México, Brasil y España, donde se identificaron las apuestas a futuro de la cadena de la mora que se convierten en apuestas directas para los actores relacionados con $\mathrm{I}+\mathrm{D}+\mathrm{i}$ para la adquisición, adopción y generación de innovaciones de producto y de proceso, así como posibles actividades de formación e investigaciones desde las universidades con enfoque agroindustrial.

Palabras claves - Agroindustria, innovación, Método Delphi, mora, prospectiva.

Abstract - This article describes the activities and results of the project "Regional Alliance for the strengthening of the Agroindustrial sector in Risaralda", in its foresight component. Delphi method was applied to consult several experts or stakeholders from the Mora sector, based on a questionnaire, which was answered anonymously in two rounds. The goal of the study is to offer to the chain value actors a tool for making decision in topics related to science, technology, and innovation. As an

Este manuscrito fue enviado el 04 de mayo de 2020 y aceptado 23 de febrero 2021. Este trabajo fue financiado por Ministerio de Educación Nacional de Colombia y la Universidad Tecnológica de Pereira mediante convenio 0898 de 2018.

L. M. Suárez-Guzmán se encuentra en la Universidad Tecnológica de Pereira, Pereira, Risaralda,Colombia (e-mail: lina.suarezguzman@utp.edu.co). important result, 26 themes in 6 thematic axes where prioritized: farming, transformation, logistics, marketing, post-harvesting, and sustainability. For the second Delphi round, nine issues were prioritized: Blackberry crop management, blackberry crop practices, record management, costs, post-harvesting, flavorings, funding sources. The participation of international experts from Mexico, Brazil, and Spain was noteworthy. This study became direct inputs to the actors of $R+D+I$ for the adoption and generation of products and innovation process in the blackberry value chain, as well as possible training and research activities in the universities with agroindutrial focus.

Index Terms - Agribusiness, blackberry, Delphi, Foresight Method, innovation

\section{INTRODUCTION}

$\mathrm{L}$ OS estudios de prospectiva tecnológica tienen una gran Iinfluencia en políticas de innovación de los países, ya que ofrece la posibilidad de identificar y aprovechar las oportunidades a futuro en las que un sector determinado debe apostar en Ciencia, Tecnología, industria y sociedad mediante un proceso participativo de científicos, industriales y gobierno [1].

En tal sentido, la prospectiva hace referencia a una herramienta que ofrece una ayuda para la aproximación de construcción de futuro, donde se plantean al menos cinco interrogantes fundamentales: ¿qué cambia?, ¿qué puede cambiar?, ¿qué debe cambiar?, ¿quién puede hacer los cambios? y ¿cuáles son las implicaciones de esos cambios [2]. La prospectiva también puede considerarse como la "Disciplina de gestión que permite reflexionar sobre el futuro - lo que podría ser y lo que debería ser -, con miras a orientar la acción humana - individual o colectiva- en el presente, mediante toda

D. A. Agudelo-Tapasco se encuentra en la Universidad Tecnológica de Pereira Risaralda ,Colombia (e-mail: diegoalejandro.agudelo@utp.edu.co)

J. W. Zartha-Sossa se encuentra en la Universidad Pontificia Bolivariana, Medellín, Colombia (e-mail: jhon.zartha@upb.edu.co)

G. L. Orozco-Mendoza se encuentra en la Universidad Pontificia Bolivariana, Medellín, Colombia (e-mail: gina.orozco@upb.edu.co) 
una infraestructura conceptual y metodológica aplicable a problemas de diferentes campos y áreas [3]."

Diversos estudios que se han realizado en Colombia de prospectiva han tenido como objetivo la identificación de las necesidades futuras de los recursos humanos que surgirá del impacto de los avances tecnológicos y las tendencias organizacionales entre los 5 y 10 años [4].

Para el caso de Risaralda, la Universidad Tecnológica de Pereira y el Ministerio de Educación Nacional unieron esfuerzos para conformar alianzas para el fortalecimiento del sector agroindustrial, donde se realizó un estudio de prospectiva a 2032 a través del método Delphi, tomando como base las necesidades del departamento de Risaralda para la consolidación como territorio de vocación agrícola sostenible. El objetivo del estudio fue "priorizar las tecnologías, innovaciones y nuevos negocios relacionados con la Mora al 2032".

El presente artículo se divide en cuatro secciones, la primera presenta un contexto enfocado en el sector agroindustrial de la Mora y el método Delphi; la segunda parte muestra la metodología llevada a cabo teniendo en cuenta la construcción inicial de un árbol temático y tecnológico, hasta la aplicación de dos rondas Delphi con su respectivo análisis estadístico, la tercera parte, presenta los resultados obtenidos clasificados en

6 agrupadores: Cultivo, Transformación, Logística, Comercialización, Poscosecha y Sostenibilidad. Al final se presentan las conclusiones del estudio.

\section{CONTEXTO}

La Mora es originaria de Centro América y crece en todo el trópico americano; aunque se han reportado especies similares en África. Para el caso de Colombia, la variedad de Mora de Castilla es la más cultivada por su adaptabilidad a las variaciones climáticas y de niveles de humedad.

TABLA I

INDICADORES DE PRODUCCIÓN (NACIONAL)

\begin{tabular}{cccccccccc}
\hline VARIABLE & $\mathbf{2 0 1 0}$ & $\mathbf{2 0 1 1}$ & $\mathbf{2 0 1 2}$ & $\mathbf{2 0 1 3}$ & $\mathbf{2 0 1 4}$ & $\mathbf{2 0 1 5}$ & $\mathbf{2 0 1 6}$ & $\mathbf{2 0 1 7}$ & $\begin{array}{c}\text { AUMENT } \\
\mathbf{0}\end{array}$ \\
\hline $\begin{array}{c}\text { AREA } \\
\text { Sembrad } \\
\text { a(Has) }\end{array}$ & 14.049 & 13.747 & 13.810 & 14.160 & 14.414 & 15.144 & 14.519 & 16.851 & $18 \%$ \\
$\begin{array}{c}\text { AREA } \\
\text { Cosechad } \\
\text { a(Has) }\end{array}$ & 12.203 & 11.651 & 11.931 & 12.006 & 12.528 & 13.072 & 12.441 & 13.882 & $14 \%$ \\
$\begin{array}{c}\text { Producció } \\
\text { n } \\
\text { (Ton) }\end{array}$ & 99.182 & 94.325 & 100.073 & 105.445 & 105.812 & 108.845 & 110.453 & 130.672 & $32 \%$ \\
$\begin{array}{c}\text { Rendimiento } \\
\text { (Ton/Has) }\end{array}$ & 8,13 & 8,1 & 8,39 & 8,78 & 8,45 & 8,33 & 8,88 & 9,41 & \\
\hline
\end{tabular}

Fuente: MADR, 2018

Teniendo en cuenta la Tabla I, entre el periodo de 2010 y 2017 el área Sembrada en cultivos de Mora en Colombia aumentó en $18 \%$, alcanzando para el último año las 16.851 hectáreas. Sin embargo, en el país, en promedio se deja de cosechar cerca de 2.000 hectáreas anuales (cerca del 12\%), que representan un potencial de 18,000 toneladas de Mora. Durante el mismo periodo la Producción Nacional presentó un aumento del $32 \%$, producto de un aumento generalizado de las siembras [6].

$\mathrm{Al}$ aumento de la producción aportaron especialmente los departamentos de Caldas, que pasó de 2.696 en 2010 a 6.019 toneladas en 2017; Santander, que pasó de 17.378 a 19.725;
Tolima, que pasó de 3548 a 7.393 toneladas producidas en 2017; Nariño, que pasó de 2.729 en 2010 a 9.707 toneladas anuales en 2017 [6].

En Risaralda, el área sembrada de mora fue de 396 hectáreas en el 2017, lo cual se traduce en una participación de $2.3 \%$ del área total del país; el área cosechada fueron 275 hectáreas, alcanzando un rendimiento correspondiente a 7 toneladas por hectárea; un panorama diferente a las demás cadenas donde el rendimiento departamental supera el promedio nacional [7].

De acuerdo a Risaralda Territorio Agroindustrial [7], donde se relaciona Área Sembrada, Área Cosechada, Producción y Rendimiento del Cultivo de Mora en los diferentes municipios de Risaralda para el año 2017, se destaca el municipio de Apia con mayor área sembrada con 88 hectáreas, el municipio de Belén de Umbría con un rendimiento de 20T/Ha y el municipio de Santa Rosa con el mayor potencial de siembra con 8,987 hectáreas. Adicionalmente, las áreas sembradas en el sector mora en el departamento de Risaralda han reducido sus cifras en los últimos 5 años, pasando de 546 hectáreas en el 2010 a 279 hectáreas en el 2017 [8]. Se produjeron 2.819 toneladas de mora en 671 fincas que conforman el aprovisionamiento de la fruta, a un precio de $\$ 1.474$ por kilogramo. El área potencial es de 19.232 hectáreas de las que se aprovechan un 2\%; las cifras de exportaciones en el departamento registran 45 toneladas vendidas al resto del mundo lo que representa una participación muy baja del departamento en las compras internacionales [7], evidenciando una crisis en la cadena a causa de los bajos precios que tiene este producto y de igual forma a la falta de aliados comerciales.

Sin embargo, a nivel alimentario la mora ha ganado posicionamiento en la industria debido a la presencia de sustancias con propiedades funcionales como los compuestos fenólicos asociados a diferentes beneficios para la salud frente a enfermedades cardiovasculares, cáncer, diabetes, procesos inflamatorios, por lo que su consuno no solo en fresco sino jugos, mermeladas, gelatina, jarabes, dulces y vino la convierten en un potencial para el mercado nacional e internacional [9]. Por tal motivo, para el estudio de prospectiva a 2032 de la cadena agroindustrial de mora se identificaron 6 agrupaciones en las cuales la cadena debe trabajar: cultivo, transformación, logística, comercialización, poscosecha y sostenibilidad para facilitar la toma de decisiones hacia las apuestas del sector.

\section{A. Método DELPHI}

El método Delphi consiste en una consulta estructurada, anónima y reiterativa a expertos, con el objeto de identificar opciones, obtener consenso y validar resultados [10]. Existen dos tipos de elementos para identificar el futuro, el exploratorio que busca identificar escenarios futuros, y los estudios normativos, que están dirigidas a priorizar un listado de temas o tecnologías genéricas, con la posibilidad de agregar a los temas o tecnologías inicialmente propuestas y otras que considere el experto que puedan hacer falta [11].

El Delphi es uno de los métodos más reconocidos y utilizados 
a nivel empresarial y académico, y su aplicación busca ayudar a reducir la incertidumbre en la toma de decisiones, y a su vez contribuir como lo explica [3] a "anticipar y actuar a través de la apropiación".

La producción científica de estudios de caso sobre el método Delphi es muy amplia, en los últimos años se cuenta con estudios como en [12], donde se menciona el uso del Delphi con la participación de profesionales y no solamente de expertos académicos, además, en [13], trabajaron a cerca de elementos territoriales o geográficos por lo que utilizaron un Delphi Espacial.

En [14], el método Delphi consistió en una consulta a un número de expertos de los sectores o temas específicos elegidos, sobre la base de un cuestionario, preparado por paneles o comisiones de expertos, que se responde anónimamente y en dos o más rondas.

Esta metodología se apoya en la selección de un grupo de expertos a los que se les pregunta su opinión sobre cuestiones referidas a acontecimientos del futuro [15]. La calidad de los resultados depende del cuidado que se ponga en la elaboración del cuestionario y en la elección de los expertos consultados [16]. Las estimaciones de los expertos se realizan en sucesivas rondas, anónimas, con el fin de conseguir consenso, pero respetando la opinión y autonomía de los participantes [17].

El método Delphi permite tener juicios grupales después de rondas sucesivas que brindan aspectos, justificaciones más efectivas que si se tuvieran juicios individuales [18]. Por otra parte, se hace una retroalimentación a las respuestas en cada ronda, con el fin de proporcionar la información suficiente para la siguiente ronda. Además, las respuestas se analizan con procedimientos estadísticos descriptivos, con el fin de llegar a un consenso [19].

\section{METODOLOGÍA}

Se realizó una consulta estructurada, anónima y reiterativa a expertos nacionales e internacionales en temas afines al sector de mora, los cuales tuvieron los siguientes agrupadores: cultivo, transformación, logística, comercialización, poscosecha, sostenibilidad al 2032. El cuestionario se dirigió a priorizar un listado de temas correspondientes a cada agrupador. La invitación con el cuestionario de la primera ronda Delphi, fue enviada a 100 expertos nacionales e internacionales de empresas del sector productivo, universidades, gobierno, sector público y privado, entre otras organizaciones del sector de Mora.

Las actividades realizadas durante el estudio de prospectiva y los temas y decisiones medulares en los talleres realizados fueron construidos y validados por docentes investigadores de la Universidad Tecnológica de Pereira - UTP y posteriormente socializados, tal como se detalla en la Fig. 1.

\begin{tabular}{|c|c|c|}
\hline FASE I & FASE II & FASE III \\
\hline $\begin{array}{l}\text { Revisión de } \\
\text { antecedentes, } \\
\text { análisis de reportes } \\
\text { de Vigilancia } \\
\text { Tecnológica del } \\
\text { sector Mora. } \\
\text { Construcción perfil } \\
\text { de expertos - } \\
\text { Elaboración de la } \\
\text { lista de expertos. } \\
\text { Construcción del } \\
\text { árbol temático y } \\
\text { tecnológico. }\end{array}$ & $\begin{array}{l}\text { Elaboración } \\
\text { cuestionario de la } \\
\text { primera ronda a } \\
100 \text { expertos. } \\
\text { Análisis estadístico } \\
\text { primera ronda } \\
\text { Delphi e Informe } \\
\text { ejecutivo. }\end{array}$ & $\begin{array}{l}\text { Elaboración } \\
\text { cuestionario } \\
\text { segunda ronda-- } \\
\text { Envío de la } \\
\text { segunda ronda. } \\
\text { Análisis } \\
\text { estadístico de la } \\
\text { segunda ronda. } \\
\text { Propuesta de } \\
\text { tecnologías, } \\
\text { innovaciones y } \\
\text { temas prioritarios } \\
\text { en el sector mora. }\end{array}$ \\
\hline
\end{tabular}

Fig. 1. Metodología aplicada

Fase I: esta etapa comprendió la revisión de antecedentes, mora, análisis de documentos de vigilancia tecnológica del sector con el fin de extraer variables que se convirtieron en insumo del árbol temático y tecnológico.

Esta etapa también comprendió la construcción del árbol temático, tecnológico. Este árbol tecnológico hace referencia al conglomerado de temas resultantes de la vigilancia tecnológica realizada en bases de datos científicas.

En esta fase también se realizó la construcción del perfil de expertos y revisión de la lista de expertos, se tuvo en cuenta que existiera un equilibrio en cuanto a expertos de universidades, empresas, estado y entidades de interfaz tales como: centros de desarrollo tecnológico (CDTs), centros de productividad, incubadoras de empresas y parques científicos y tecnológicos entre otros.

Fase II: en esta fase se llevó a cabo la elaboración del cuestionario para la primera ronda Delphi, se realizó con base en el árbol de temas y tecnologías para cada agrupador, los expertos contestaron la importancia o prioridad de cada tema o tecnología a 2032 en escala liker de 0 a 5, donde 5 es la más alta prioridad, en esta ronda se solicitó a los participantes anexar comentarios o justificaciones para los temas y tecnologías.

Una vez recibida la primera ronda Delphi debidamente diligenciada, se procedió a realizar el análisis estadístico donde se utiliza diferentes técnicas estadísticas tales como: moda, frecuencia modal, porcentaje de consenso, técnicas que han sido referenciadas en estudios previos [20].

Fase III: en esta fase se realizó la construcción y envío de la segunda ronda Delphi, la cual buscaba retroalimentar los resultados obtenidos en la primera ronda, debido a que en esta etapa se le solicitó al experto que al momento de diligenciar la encuesta que "suba" los temas y tecnología en discusión al grupo de prioritarios y que "baje" aquellos que ve en prioritarios pero que considera que deberían estar en el grupo de discusión. Se aplicaron dos rondas de acuerdo con las tendencias de reducción del número de rondas y a los resultados de estudios previos realizados por [21] quienes hablan de la gestión sostenible en programas de cría de ganado en los países en desarrollo [22], se examinaron los enfoques de desarrollo de capacidades para la gestión de la propiedad intelectual (PI) en investigación y desarrollo agrícola (I + D), con [23], se identificaron las alternativas de manejo y varias características forestales en la preservación de la biodiversidad y los hábitats 
en la zona boreal de los países nórdicos y otras investigaciones en agroindustria [24].

Después de recibir la segunda ronda Delphi debidamente diligenciada, se procedió a realizar el análisis estadístico donde se utiliza las técnicas estadísticas descritas para la primera ronda, en esta etapa, se presentan los resultados finales de la aplicación del método Delphi, donde se presentan luego de las 2 rondas Delphi los agrupadores con sus respectivos temas prioritarios.

\section{RESUlTADOS}

Para la elaboración de la primera ronda Delphi se presentaron a los expertos un total de 73 temas en 6 agrupadores de la cadena de mora: Cultivo, transformación, logística, comercialización, poscosecha y sostenibilidad, los cuales se describen a continuación y fueron incluidos en el formato enviado.

Después de que el experto diligenció la información inicial sobre afiliación y nivel de formación, contestó el cuestionario con los temas por cada agrupador:

Grupo 1, Cultivo: Variedades de semillas para siembra, Organizaciones de productores, Recurso humano capacitado (técnicos), Asistencia técnica, Manejo sanitario, Manejo fitosanitario, Mejoramiento genético, Citogenética, Calidad en los insumos, Inocuidad en los insumos, Material de siembra, Diferenciación morfológica, Perfil aromático, Cultivos meristemáticos, Cultivos microsatelites, Cultivos micropropagación, Control de plagas (micorrización), Acceso a sistemas de información, Zonificación, Georreferenciación, Socio economía, Planeación de la producción.

Grupo 2, Transformación: Pulpas, Alimentos mínimamente procesados, empleo de mora como sustrato para bioprocesos, deshidratación, refrigeración, secado por pulverización, microencapsulación, liofilización, medios ultrasónicos, IV gama, empaques especializados (mantener características fisicoquímicas), modelos de producción (análisis por redes neuronales), Producción de jugos, Producción de jaleas, Producción de licores, Producción de bebidas con probióticos, Biofabricación de nanopartículas de óxido de cobre a partir del tratamiento de nitrato de cobre con la mora, Empaques de atmósfera modificada, Impregnación al vacío, Nanopartículas metálicas de fuentes biológicas, Transferencia de tecnología, Ultracongelación.

Grupo 3, logística: Vías de acceso, Transporte, Intermediarios, Almacenamiento, Empaques-Embalaje, Cadena de frio.

Grupo 4, comercialización: Nuevos productos (alimentarios y no alimentarios), Asociatividad, Exportación, Importación, Apertura a nuevos mercados, Investigación de mercados, Alianza productores/Agroindustria/mercado en fresco, Fuentes de financiación (incentivos), Reducción de costos.

Grupo 5, poscosecha: Asociaciones productoras, Maquinaria y equipos, Conservación, Empaques, Acopio, Transporte, Manipulación, Buenas prácticas agrícolas, Transferencia de tecnología.

Grupo 6, Sostenibilidad: Producción en sistemas agroecológicos, Medio ambiente, Producción limpia, Tecnologías emergentes, Mano de obra calificada.

Después de cerrar la fecha de recepción de cuestionarios de la primera ronda, se recibieron un total de 21 respuestas de pares académicos expertos en mora, como se muestra en la Fig. 2, sobre las cuales se aplicó un análisis estadístico que incluyó el cálculo de la de moda, la frecuencia modal, el porcentaje de consenso con los temas prioritarios, no prioritarios y en discusión.

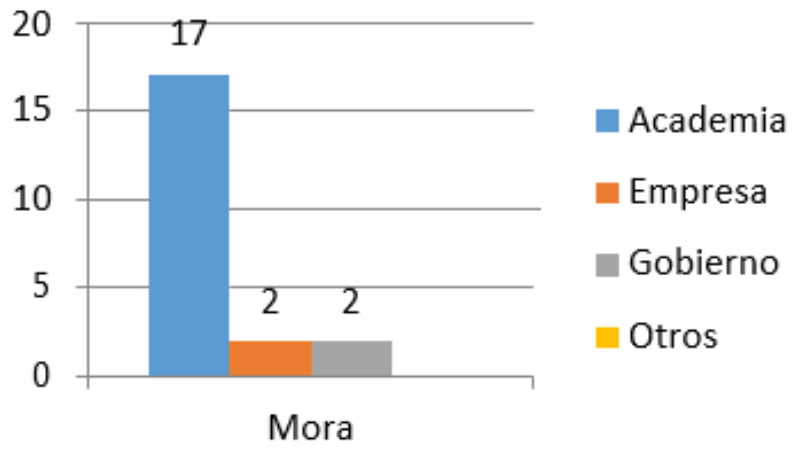

Fig. 2. Nivel de formación académica de los expertos

En cuanto a la afiliación de los expertos que participaron en el estudio se contó con la participación de 17 académicos, 2 empresarios y 2 representantes de instituciones públicas; dentro del grupo se contó con aportes de 2 expertos de México, 1 de Brasil y 1 de España.

Después de los análisis estadísticos realizados de acuerdo con el método mencionado en la metodología, se hallaron:

Temas prioritarios: 26 Temas no prioritarios: 0 Temas en discusión: 43

Temas No Sabe / No Responde: 4 Temas Nuevos: 9

En la Fig. 3, se muestran los porcentajes de temas prioritarios por cada agrupador:

\section{Temas Prioritarios}
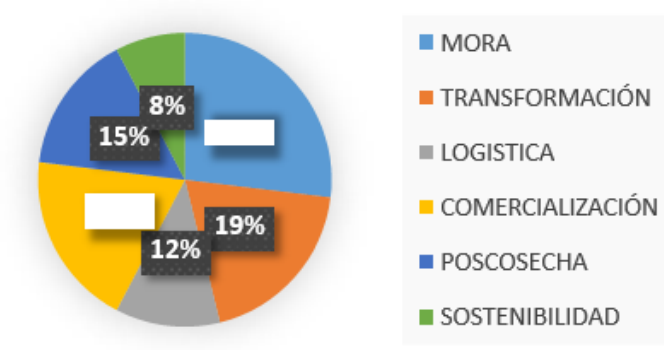

Fig. 3. Porcentaje de temas prioritarios

En los temas prioritarios se obtuvo que el $27 \%$ requiere concentrar esfuerzos para fortalecer todo lo relacionado con el cultivo; el 19\% en Transformación (Agroindustria), el 18\% en comercialización y $15 \%$ en poscosecha.

Los 9 temas nuevos de la primera ronda fueron: Manejo del cultivo de mora, prácticas de manejo del cultivo de mora, manejo de registros, costos de producción, producción 
(orgánica o limpia), poscosecha (momento de cosecha), colorantes, saborizantes, fuentes de financiamiento.

Con base en lo anterior, se procedió a elaborar el informe ejecutivo de la primera ronda Delphi. Este fue un insumo fundamental para los expertos que contestaron la segunda ronda, ya que una de las características del método Delphi es la retroalimentación para el experto, el número de justificacioneso retroalimentaciones obtenidas en la primera ronda fue de 146, es decir entre 6 y 7 justificaciones por experto.

La construcción del cuestionario de segunda ronda Delphi, se realizó con base en el resultado de la primera ronda. En las instrucciones se presentó una explicación de cómo contestar cada agrupador, ya que los cambios que el experto realizó eran diferentes para cada agrupador. Básicamente el experto tuvo que leer los aspectos relevantes que en la primera ronda se consolidaron para cada tema de cada agrupador. Con base en la información suministrada, el experto pudo establecer sí un ítem que estaba en prioritario, debería pasar a discusión, y de los temas en discusión, debería pasar a prioritario.

Después de cerrar la recepción de respuestas, se procedió a realizar el análisis estadístico donde se utilizaron las técnicas estadísticas descritas para la primera ronda, además, se calculó la frecuencia modal 2 (Fm2) la cual contiene la frecuencia modal 1(Fm1) más el número de expertos que "entran" el tema

o tecnología en la ronda dos (pero que en la ronda 1 no lo habían calificado como prioritario) menos los expertos que "sacan" el tema o tecnología en la ronda dos (pero que en la ronda uno lo habían calificado como prioritario), permitiendo de esta forma obtener los temas prioritarios.

En la Tabla II, se observan los resultados obtenidos del análisis de la segunda ronda Delphi en el sector mora en comparación con la primera ronda Delphi.

TABLA II.

RESumen De Temas Y TeCnOlogías PRIORITARIOS En Las Dos Rondas DELPHI

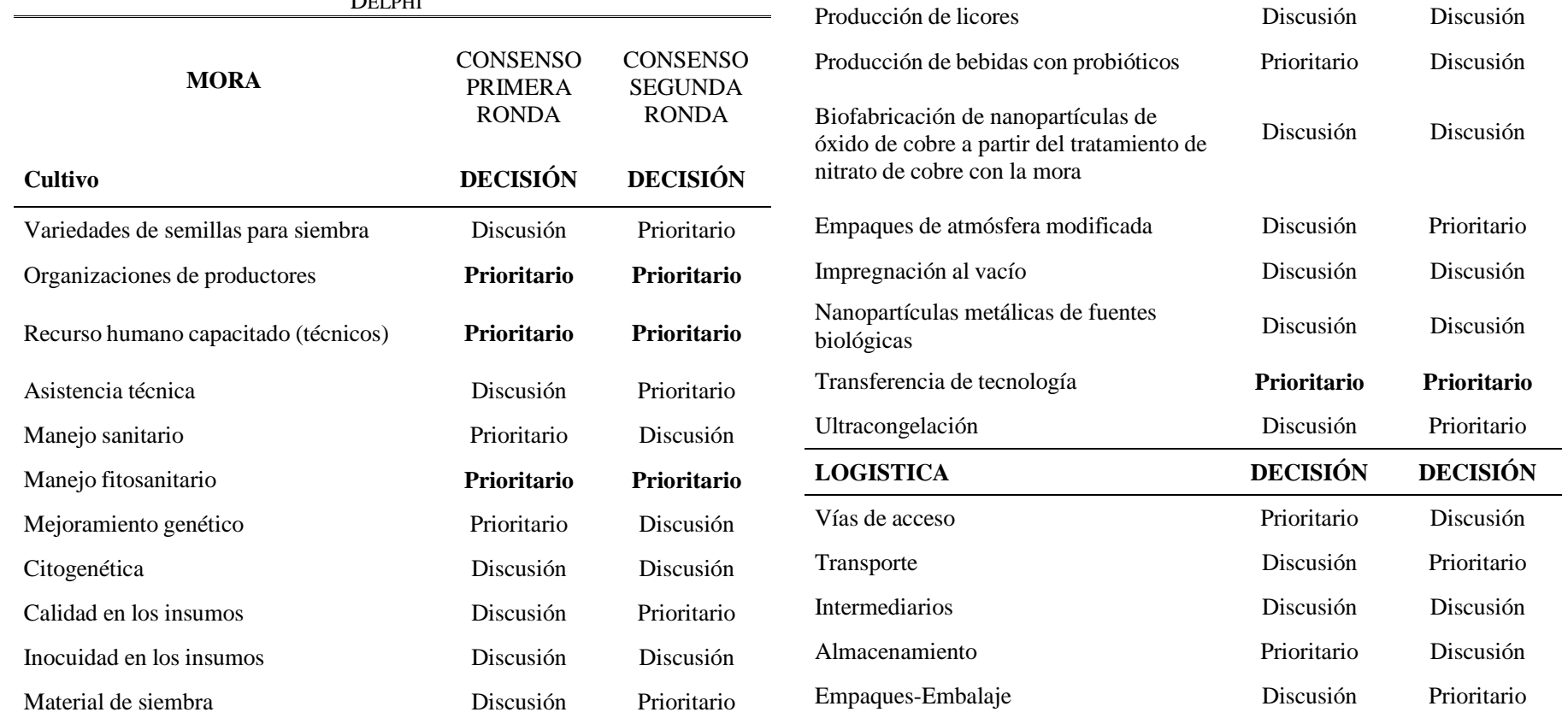

\begin{tabular}{|c|c|c|}
\hline Diferenciación morfológica & Discusión & Discusión \\
\hline Perfil aromático & Discusión & Discusión \\
\hline Cultivos meristemáticos & Prioritario & Discusión \\
\hline Cultivos microsatélites & Discusión & Discusión \\
\hline Cultivos micropropagación & Prioritario & Discusión \\
\hline Control de plagas (micorrización) & Discusión & Discusión \\
\hline Acceso a sistemas de información & Discusión & Prioritario \\
\hline Zonificación & Discusión & Prioritario \\
\hline Georreferenciación & Discusión & Discusión \\
\hline Socioeconomía & Discusión & Discusión \\
\hline Planeación de la producción & Discusión & Prioritario \\
\hline TRANSFORMACIÓN & DECISIÓN & DECISIÓN \\
\hline Pulpas & Discusión & Discusión \\
\hline Alimentos mínimamente procesados & Prioritario & Discusión \\
\hline $\begin{array}{l}\text { Empleo de mora como sustrato para } \\
\text { bioprocesos }\end{array}$ & Discusión & Prioritario \\
\hline Deshidratación & Discusión & Discusión \\
\hline Refrigeración & Discusión & Prioritario \\
\hline Secado por pulverización & Discusión & Prioritario \\
\hline Microencapsulación & Discusión & Discusión \\
\hline Liofilización & Discusión & Discusión \\
\hline Medios ultrasónicos & Discusión & Discusión \\
\hline IV Gama & Discusión & Prioritario \\
\hline $\begin{array}{l}\text { Empaques especializados (mantener } \\
\text { características fisicoquímicas) }\end{array}$ & Prioritario & Prioritario \\
\hline $\begin{array}{l}\text { Modelos de producción (análisis por } \\
\text { redes neuronales) }\end{array}$ & Discusión & Discusión \\
\hline Producción de jugos & Prioritario & Discusión \\
\hline Producción de jaleas & Discusión & Discusión \\
\hline Producción de licores & Discusión & Discusión \\
\hline Producción de bebidas con probióticos & Prioritario & Discusión \\
\hline $\begin{array}{l}\text { Biofabricación de nanopartículas de } \\
\text { óxido de cobre a partir del tratamiento de } \\
\text { nitrato de cobre con la mora }\end{array}$ & Discusión & Discusión \\
\hline Empaques de atmósfera modificada & Discusión & Prioritario \\
\hline Impregnación al vacío & Discusión & Discusión \\
\hline $\begin{array}{l}\text { Nanopartículas metálicas de fuentes } \\
\text { biológicas }\end{array}$ & Discusión & Discusión \\
\hline Transferencia de tecnología & Prioritario & Prioritario \\
\hline Ultracongelación & Discusión & Prioritario \\
\hline LOGISTICA & DECISIÓN & DECISIÓN \\
\hline Vías de acceso & Prioritario & Discusión \\
\hline Transporte & Discusión & Prioritario \\
\hline Intermediarios & Discusión & Discusión \\
\hline Almacenamiento & Prioritario & Discusión \\
\hline Empaques-Embalaje & Discusión & Prioritario \\
\hline
\end{tabular}




\begin{tabular}{lcc} 
Cadena de frio & Prioritario & Discusión \\
\hline COMERCIALIZACIÓN & DECISIÓN & DECISIÓN \\
\hline $\begin{array}{l}\text { Nuevos productos (alimentarios y no } \\
\text { alimentarios) }\end{array}$ & Prioritario & Discusión \\
Asociatividad & Prioritario & Discusión \\
Exportación & Discusión & Prioritario \\
Importación & Discusión & No Prioritario \\
Apertura a nuevos mercados & Prioritario & Discusión \\
Investigación de mercados & Prioritario & Discusión \\
Alianza & & \\
productores/Agroindustria/mercado en & Prioritario & Prioritario \\
fresco & & \\
Fuentes de financiación (incentivos) & Discusión & Prioritario \\
Reducción de costos & Discusión & Prioritario \\
\hline POSCOSECHA & DECISIÓN & DECISIÓN \\
\hline Asociaciones productoras & Prioritario & Discusión \\
Maquinaria y equipos & Discusión & Discusión \\
Conservación & Discusión & Prioritario \\
Empaques & Prioritario & Discusión \\
Acopio & Discusión & Prioritario \\
Transporte & Discusión & Prioritario \\
Manipulación & Discusión & Prioritario \\
Buenas prácticas agrícolas & Prioritario & Prioritario \\
Transferencia de tecnología & Prioritario & Discusión \\
\hline SOSTENIBILIDAD & DECISIÓN & DECISIÓN \\
\hline Producción en sistemas agroecológicos & Prioritario & Discusión \\
Medio ambiente & Discusión & Prioritario \\
Producción limpia & Prioritario \\
\hline
\end{tabular}

De acuerdo con la tabla anterior se obtuvieron 26 temas y tecnologías prioritarias en la primera ronda de los cuales 32 de ellos cumplieron con el requisito de ser prioritarios en la segunda ronda. En el grupo uno de variables sobre Cultivo, 3 temas y tecnologías fueron prioritarios en las dos rondas. Los temas y tecnologías que cumplieron esta característica fueron: Organizaciones de productores, recurso humano capacitado (técnicos) y manejo fitosanitario; mientras que 7 temas que no fueron prioritarios en la primera ronda, si entró en la categoría en la segunda.

En el grupo 2 transformación, 5 temas y tecnologías fueron prioritarias en la primera ronda, mientras que 8 fueron prioritarios en la segunda ronda; los que entraron en la categoría de prioritarios en la primera y segunda ronda fueron: empaques especializados (mantener características fisicoquímicas) y transferencia de tecnología.

En el grupo 3 logísticas, 3 temas y tecnologías fueron prioritarias en la primera ronda, las cuales se mencionan a continuación: vías de acceso, almacenamiento y cadena de frío; mientras que 2 no fueron prioritarios en la primera ronda, pero si entraron como prioritarios en la segunda: transporte y empaques - embalaje.

En el grupo 4 comercialización, 5 temas y tecnologías fueron prioritarios en la primera ronda, mientras que 3 no fueron prioritarios en la primera ronda, pero entraron como prioritarios en la segunda ronda. El tema que fue prioritarios en ambas fue, Alianza productores/Agroindustria/mercado en fresco.

En el grupo 5 poscosecha, un tema y tecnología fue prioritario en la primera y segunda ronda: buenas prácticas agrícolas; 3 temas fueron priorizados en la primera ronda y 4 que no fueron priorizados en la primera, lo fueron en la segunda.

En el grupo 6 sostenibilidad, un tema y tecnología fue prioritario en la primera ronda, mientras que 2 fueron prioritarios en la segunda ronda; el que entró en la categoría de prioritarios en la primera y segunda ronda fue: producción limpia.

\section{DISCUSIÓN}

Un análisis adicional importante es el relacionado con los temas, tecnologías e innovaciones que en la primera ronda quedó en discusión, pero que en la segunda ronda lograron aumentar su consenso pasando al grupo de prioritarios.

A continuación, se observa en la Tabla III los nuevos temas prioritarios de la 2da ronda Delphi:

TABLA III

TEMAS PRIORITARIOS EN LA 2DA RONDA DELPHI

\begin{tabular}{ll}
\hline \hline & \multicolumn{1}{c}{ Temas prioritarios } \\
\hline 1 & Variedades de semillas para siembra \\
2 & Asistencia Técnica \\
3 & Calidad en los insumos \\
4 & Asistencia Técnica \\
5 & Acceso a sistemas de información \\
6 & Zonificación \\
7 & Planeación de la Producción \\
8 & Empleo de mora como sustrato para bioprocesos \\
9 & Refrigeración \\
10 & Secado por pulverización \\
11 & IV Gama \\
12 & Empaques de atmósfera modificada \\
13 & Ultracongelación \\
14 & Transporte \\
15 & Empaques - embalaje \\
16 & Exportación \\
17 & Fuentes de financiación (incentivos) \\
18 & Reducción de costos \\
19 & Conservación \\
20 & Acopio \\
21 & Transporte \\
22 & Manipulación \\
\hline
\end{tabular}


23

24 Tecnologías emergentes

Los temas, tecnologías e innovaciones presentados en la tabla anterior tuvieron moda alta en la primera ronda y lograron clasificación como temas en discusión, pero en la segunda ronda lograron un consenso mayor, lo que significa que los Stakeholders participantes, cambiaron sustancialmente de opinión sobre su importancia o prioridad al 2032. Estos temas complementan a los 26 temas, tecnologías e innovaciones analizados en el capítulo de resultados que fueron prioritarios en las dos rondas todos estos temas en conjunto se constituyen en insumo para universidades, grupos, centros de investigación y empresarios para la toma de decisiones frente a investigación, desarrollo, compra, venta, absorción, adopción y transferencia de tecnologías e innovaciones así como para la generación de estrategias de formación a través de cursos de capacitación que desde las universidades relacionadas con programas de agroindustria y alimentos pueden ofrecer a los productores, gremios, empresarios y otros grupos de interés relacionados con la cadena de la mora.

Teniendo en cuenta que datos publicados sobre mora, indican que puede considerarse como un alimento valioso debido a su composición y actividad biológica como minerales, vitaminas, azúcares y fitoquímicos (antocianinas y flavonoides), responsables de varios beneficios para la salud, incluyendo la prevención de la inflamación, enfermedades cardiovasculares, y el cáncer [25], estas tecnologías e innovaciones se presenten como insumo para las empresas del sector para avanzar principalmente en la formalización de variedades de mora, desarrollar mecanismos de manipulación y conservación de fruta para generar nuevos productos.

Los resultados del método Delphi se convierten en apuestas directas para los actores, ya que son insumos para los actores de la cadena para proyectos de $\mathrm{I}+\mathrm{D}+\mathrm{i}$, nuevas competencias o habilidades, adopción y transferencia de tecnología y generación de emprendimientos - Star Ups que puedan constituirse en nuevos actores del sistema sectorial de innovación.

\section{CONCLUSIONES}

El trabajo de prospectiva realizado en la cadena productiva de la Mora indican las apuestas a futuro de este producto desde lo agronómico, transformación, comercialización, innovación, son de interés para el sector, para enfrentar los obstáculos y/o brechas que se presentan y de esta forma ofrecer alternativas de solución en cada uno de los eslabones de la cadena, para que se convierta en un producto de potencial agroindustrial en el departamento, en áreas como cultivo, transformación, logística, comercialización, poscosecha y sostenibilidad.

Dentro de las áreas de estudio se agruparon 73 temas de los cuales 26 fueron prioritarios, 43 fueron temas de discusión y 9 temas nuevos en la primera ronda Delphi. Sin embargo, 32 temas y tecnologías fueron prioritarios en la segunda ronda de los cuáles, sólo 8 cumplieron con el requisito de prioritario en ambas rondas: Organizaciones de productores, recurso humano capacitado (técnicos), manejo fitosanitario, empaques especializados (mantener características fisicoquímicas), transferencia detecnología, alianza productores /Agroindustria /mercado en fresco, buenas prácticas agrícolas y producción más limpia.

En la segunda ronda Delphi 24 temas fueron priorizados por los Stakeholders participantes: que complementan los 26 que se priorizaron en la primera ronda, así mismo transporte $\mathrm{y}$ empaques y embalaje fueron priorizados en el agrupador logístico pero ningún tema fue prioritario en ambas rondas.

Los resultados obtenidos de este estudio se convierten en apuestas directas para los actores relacionados con la $\mathrm{I}+\mathrm{D}+\mathrm{i}$ ya que son insumos para la toma de decisiones frente a la investigación y desarrollo de las tecnologías prioritarias, así como para la adquisición, adopción, generación de innovaciones de producto y proceso, también son fuente de posibles actividades de formación desde las universidades, estos temas, tecnologías e innovaciones trascienden a la regiónespecifica donde se realizó el estudio y se convierten en variables estratégicas para actores relacionados con el sistema sectorial de ciencia, tecnología e innovación del subsector de la mora.

\section{AGRADECIMIENTOS}

A la Universidad Tecnológica de Pereira, al Ministerio de Educación Nacional y a la Facultad de Ciencias agrarias y Agroindustria, quienes financiaron esta investigación medianteel Convenio Interadministrativo número 0898 de 2018 suscritoentre el Ministerio de Educación Nacional y la Universidad Tecnológica de Pereira.

\section{REFERENCIAS}

[1] D. Meissner, "Results and impact of national Foresight-studies," Futures, vol. 44, no. 10, pp. 905- 913, 2012. DOI: 10.1016/j.futures.2012.07.010

[2] Cepal and Naciones Unidas, "Prospectiva y desarrollo El clima de la igualdad en América Latina y el Caribe a 2020," Publicación las Nac. Unidas, p. 65, 2013.

[3] M. Godet, "De la anticipación a la acción" en Estrategia y gestión competitiva, vol. 5, marcombo BOIXAREU editores, España, 1993, pp. 329-332.

[4] J. Wilder Zartha -Sossa, J. Manuel Montes -Hincapié,D. Toro Jaramillo, R. Hernandez -Zarta, H. Samuel Villada -Castillo, and J. Luis Hoyos -Concha, "MÉTODO DELPHI EN ESTUDIOS DE PROSPECTIVA TECNOLOGICA: Una aproximación para calcular el número de expertos y aplicación del coeficiente de competencia experta 'K," Biotecnol. en el Sect. Agropecu. y Agroindustrial, vol. 15, no. 1, pp. 105-115, 2017. DOI: 10.18684/BSAA(15)105-115

[5] K. M. Weinberger and T. A. Lumpkin, "Horticulture for Poverty Alleviation - The Unfunded Revolution," SSRN Electron. J., Dec. 2011. DOI: $10.2139 / \mathrm{ssrn} .781784$

[6] "Agronet MinAgricultura Estadísticas." [Online]. Available: https://www.agronet.gov.co/estadistica/Paginas/home. aspx?cod=1. [Accessed: 04-Mar-2020].

[7] A. Londoño-Melendez, R. Rey- Rodriguez, and M. Pérez-Holguin, "Risaralda, Territorio Agroindustrial," 1384.

[8] S. Cadena De La, M. I. Carlos, H. Escobar Torres, and E. Frutales Tropicales, "CADENA PRODUCTIVA NACIONAL DE LA MORA INDICADORES DE APOYO INDICADORES DE APOYO Cadenas Productivas," 2014.

[9] M. G. de Gomes et al., "Blackberry juice anthocyanidins limit cisplatin-induced renal pathophysiology in mice," Pathophysiology, 
vol. 26, no. 2, pp. 137-143, Jun. 2019. DOI: 10.1016/j.pathophys.2019.04.004

[10] C. C. Ramírez, J. W. Zartha, B. Arango, and G. L. Orozco, "Prospectiva 2025 de la carrera de ingeniería química en algunos países pertenecientes a la organización de estados americanos (OEA)," Form. Univ., vol. 9, no. 6, pp. 127-138, 2016. DOI: 10.4067/S0718-50062016000600012

[11] J. Steurer, "The Delphi method: An efficient procedure to generate knowledge," Skeletal Radiology, vol. 40, no. 8. pp. 959-961, Aug2011. DOI: $10.1007 / \mathrm{s} 00256-011-1145-\mathrm{z}$

[12] T. Münzberg, M. Wiens, and F. Schultmann, "A spatial-temporal vulnerability assessment to support the building of community resilience against power outage impacts," Technol. Forecast. Soc. Change, vol. 121, pp. 99-118, Aug. 2017. DOI: 10.1016/j.techfore.2016.11.027

[13] S. Di Zio, J. D. Castillo Rosas, and L. Lamelza, "Real Time Spatial Delphi: Fast convergence of experts' opinions on the territory," Technol. Forecast. Soc. Change, vol. 115, pp. 143-154, Feb. 2017. DOI: 10.1016/j.techfore.2016.09.029

[14] M. M. Castelló-Tarrega and J. Callejo, "LA PROSPECTIVA TECNOLOGICA Y SUS METODOS SECRETARIA PARA LA TECNOLOGIA, LA CIENCIA Y LA INNOVACION PRODUCTIVA," 2000.

[15] J. Nazarko et al., "Foresight Study of Road Pavement Technologies," in Procedia Engineering, 2015, vol. 122, pp. 129136. DOI: $10.1007 / \mathrm{s} 00256-011-1145-\mathrm{z}$

[16] P. D. Andersen, B. H. Jørgensen, L. Lading, and B. Rasmussen, "Sensor foresight - Technology and market," Technovation, vol. 24, no. 4 , pp. $311-320$, Apr. 2004. DOI: 10.1016/S01664972(02)00072-X

[17] T. Gnatzy, J. Warth, H. von der Gracht, and I. L. Darkow, "Validating an innovative real-time Delphi approach - A methodological comparison between real-time and conventional Delphi studies," Technol. Forecast. Soc. Change, vol. 78, no. 9, pp. 1681-1694, Nov. 2011. DOI: 10.1016/j.techfore.2011.04.006

[18] C. Santos, M. Araújo, and N. Correia, "A methodology for the identification of strategic technological competences: An application in the sheet metal equipment industry," 2017.

[19] M. A. Gomez Paz, A. Camarero Orive, and N. González Cancelas, "Use of the Delphi method to determine the constraints that affect the future size of large container ships," Marit. Policy Manag., vol. 42, no. 3, pp. 263-277, Apr. 2015. DOI: $10.1080 / 03088839.2013 .870358$

[20] J. W. Zartha Sossa, W. Halal, and R. Hernandez Zarta, "Delphi method: analysis of rounds, stakeholder and statistical indicators," Foresight, vol. 21, no. 5, pp. 525-544, Sep. 2019. DOI: 10.1108/FS11-2018-0095

[21] Y. Camara, F. Sow, B. Govoeyi, N. Moula, M. M. Sissokho, and N. Antoine-Moussiaux, "Stakeholder involvement in cattle-breeding program in developing countries: A Delphi survey," Livest. Sci., vol. 228, pp. 127-135, Oct. 2019. DOI: 10.1016/j.livsci.2019.08.014

[22] H. H. Odame, V. Henson Apollonio, and N. Thaher, "Capacity development for intellectual property management in Canadian crop biotechnology,” Int. J. Intellect. Prop. Manag., vol. 9, no. 3/4, p. 287, 2019. DOI: 10.1504/ijipm.2019.10024586

[23] A. Filyushkina, N. Strange, M. Löf, E. E. Ezebilo, and M. Boman, "Applying the Delphi method to assess impacts of forest management on biodiversity and habitat preservation," For. Ecol. Manage., vol. 409, pp. 179-189, Feb. 2018. DOI: 10.1016/j.foreco.2017.10.022

[24] J. W. Zartha, V. T. álvarez, J. C. Oviedo, and G. L. Orozco, "Proposal of a thematic and technological tree on agroindustrial engineering as a tool for a prospective study," Form. Univ., vol. 12, no. 4, pp. 13-26, 2019. DOI: 10.4067/S0718- 50062019000400013

[25] M. Schulz and J. F. Chim, "Nutritional and bioactive value of Rubus berries," Food Bioscience, vol. 31. Elsevier Ltd, p. 100438, 01-Oct2019. DOI: $10.1016 /$ j.fbio.2019.100438

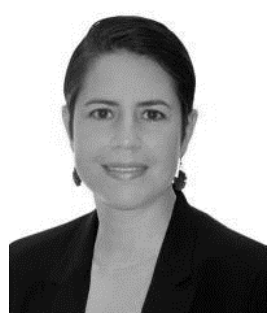

Lina María Suárez Guzmán nació en Armenia, Quindío, Colombia en 1979. Ingeniera Agroindustrial de la Universidad San Buenaventura Cali, Magister en Ciencia e Ingeniería de Alimentos de la Universidad Politécnica de Valencia. Actualmente es Decana de la Facultad de Ciencias Agrarias y Agroindustria de la Universidad

Tecnológica de Pereira.

ORCID: https://orcid.org/0000-0002-8758-3098

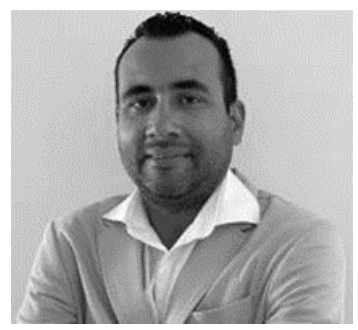

Diego Alejandro Agudelo Tapasco, nació en Santa Rosa de Cabal, Risaralda, Colombia en 1978. Ingeniero Ambiental de la Universidad Libre Seccional Pereira, Agrónomo de la Universidad Nacional Abierta y a Distancia UNAD y Especialista en Procesos Industriales Agroalimentarios de la Universidad Tecnológica de Pereira. Profesor y profesional de apoyo a proyectos de la Facultad de Ciencias Agrarias y Agroindustria de La Universidad Tecnológica de Pereira.

RCID: https://orcid.org/0000-0001-7481-2819

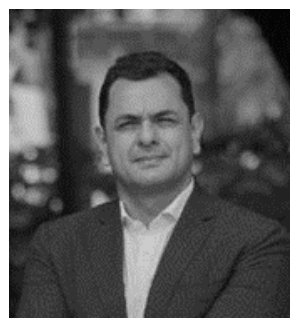

Jhon Wilder Zartha Sossa nació en Armenia, Quindío, Colombia en 1971. Ingeniero Agroindustrial de la Universidad La Gran Colombia de Armenia, Magíster en Gestión Tecnológica de la Universidad Pontificia Bolivariana y Doctor en Administración de la Universidad de Medellín. Profesor Titular de la UPB e investigador Senior en Colciencias.

ORCID: https://orcid.org/0000-0001-7766-2780

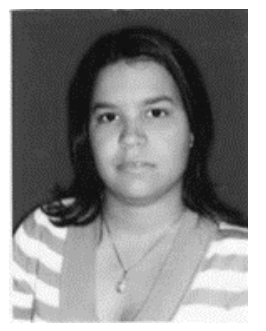

Gina Lía Orozco Mendoza nació en San Juan del Cesar, Colombia en 1979. Ingeniería Agroindustrial de la Universidad Pontificia Bolivariana, Especialista en Gestión de la Innovación Tecnológica y Magister en Gestión Tecnológica de la Universidad Pontificia Bolivariana. Coordinadora de formación avanzada de la escuela de Ingenierías de la Universidad Pontificia Bolivariana y coordinadora académica de la maestría en sostenibilidad.

ORCID: https://orcid.org/0000-0003-2914-0683 